\title{
La calidad de vida desde el enfoque de las capacidades en la crisis de la COVID-19 en Perú
}

\author{
Franklin Cornejo Urbina \\ Universidad Antonio Ruiz de Montoya
}

\section{Resumen}

El presente trabajo propone algunas conexiones teóricas sobre el enfoque de las capacidades para repensar el concepto de calidad de vida en la pandemia de la COVID-19. En particular, este análisis se proyecta hacia los contextos peruanos de desigualdad social y pobreza, donde la agencia y las acciones colectivas -como potencialidades significativas de las personas- pueden promover capacidades frente a la crisis ocasionada por la pandemia. Se concluye que las personas organizadas, en contextos de vulnerabilidad social se identifican valores comunes, como la democracia, la justicia o la verdad: se defienden valores constitutivos para tener calidad de vida y desarrollo humano.

Palabras clave: calidad de vida, enfoque de las capacidades, COVID-19, desigualdad social, pobreza.

\begin{abstract}
:
The present work proposes some theoretical connections on the capabilities approach to rethink the concept of quality of life in the COVID-19 pandemic. In particular this analysis is projected towards the Peruvian contexts of social inequality and poverty, where the agency and the collective actions -as significant potentialities of people- can promote capacities towards to the crisis caused by the pandemic. It is concluded that organized people, in contexts of social vulnerability, identify common values such as democracy, justice or truth: they defend constitutive values to have quality of live and human development.
\end{abstract}

Key words: quality of life, capabilities approach, COVID-19, social inequality, poverty. 


\section{Introducción. Una teoría social inclusiva}

En el Perú, la pandemia de la COVID-19 ha desatado discusiones en los ámbitos político y mediático sobre la baja inversión del Estado en salud, también en lo relacionado a la informalidad, el desempleo y el insuficiente apoyo a la población en situación de pobreza. Una de las principales críticas es que el Estado en Perú no ha generado condiciones estructurales para que la población acceda a servicios públicos básicos y al bienestar social. Se cuestiona el hecho de que un país con crecimiento económico sostenido en 20 años no se haya especializado en atender a sus ciudadanos, lo cual, a su vez, ha ampliado las brechas de desigualdad existentes ${ }^{1}$. Ante la crisis de la COVID-19, ¿`cómo se pueden replantear los conceptos de calidad de vida y desarrollo humano en contextos de desigualdad? ¿Qué aspectos del enfoque de las capacidades permiten ampliar la comprensión de la agenda social de los pobladores peruanos en situación de vulnerabilidad social?

Este texto plantea que la comprensión y puesta en práctica de capacidades, entendidas como "libertades que tienen las personas para elegir y actuar” (Nussbaum, 2012), implica la realización de "funcionamientos", que permiten lograr una calidad de vida, como el hecho de tener salud, recibir educación, participar de la vida pública y política, o formar parte de una comunidad, entre otras capacidades que la investigadora Martha Nussbaum ha organizado en una lista de diez "capacidades centrales" relacionadas con tener una vida digna y bienestar. En consecuencia, la falta de capacidades y funcionamientos que tienen las personas en la crisis de la pandemia que azota al Perú ha movilizado a personas, familias y organizaciones para autoorganizarse y activar redes de solidaridad. Sin embargo, estas medidas son temporales y paliativas, y no representan soluciones definitivas a los problemas. Es así como la agencia de las personas y las acciones colectivas se presentan como capacidades significativas para que las

1 Según el Banco Mundial, la pandemia de la COVID-19 ha ocasionado que "la pérdida de empleos y fuentes de ingreso sea bastante alta en el Perú y más pronunciada aún en los sectores informales, independientes y con bajo nivel educativo de la población”. 
personas afronten de manera individual o comunitaria las dificultades y las brechas que experimentan en sus contextos y culturas. Las libertades y los funcionamientos entendidos también como valores asumidos de manera individual y compartida socialmente están condicionados al desarrollo y a una democracia sólida. Según Sen (2000), "el desarrollo y el fortalecimiento de un sistema democrático constituye un componente esencial del proceso de desarrollo [...]. La importancia de la democracia reside en tres virtudes: 1) su importancia intrinseca, 2) su contribución instrumental, y 3) su papel constructivo en la creación de valores y normas. Ninguna evaluación del sistema democrático de gobierno puede estar completa si no se considera cada una de ellas por separado" (p. 197).

Se propone que mientras se tenga conciencia y se racionalicen los valores relacionados con las capacidades de agencia y acción colectiva (y allí la importancia de tener valores y principios para esa reflexión y ese diálogo), entre el Estado, las instituciones y las personas, se logrará que las dos últimas perciban de manera positiva la vida en relación con su entorno familiar y ciudadano, estableciendo relaciones de confianza, respeto y servicio. Estas capacidades, en un contexto de crisis y emergencia sanitaria, son de urgente atención humanitaria y ciudadana. Desde esta perspectiva, se presenta una revisión al enfoque de las capacidades para redimensionar el concepto de calidad de vida en la crisis de la pandemia; y en una búsqueda posible de definiciones para destacar la participación de las personas en la sociedad, la consolidación de la actoría ciudadana y re-encontrar el papel del Estado como garante de derechos y libertades.

Se analizan abordajes posibles relacionados al enfoque de las capacidades; luego, se destaca la acción colectiva y la actoría social como factores claves para contrarrestar la crisis de representación política, cuyo efecto extremo es la desatención del Estado o su ausencia en un modelo de Estado democrático. Sin libertades ni democracia, vistas como capacidades percibidas y experimentadas por las personas, la calidad de vida presenta serias limitaciones para realizarse. Ello implica la existencia de problemas para que se pueda dar en las personas, tal como lo indica Ardila (2003, p. 163): "un estado de satisfacción general, 
que surge de realizar las potencialidades que el individuo posee", pero al mismo tiempo el hecho de "tener relaciones armónicas con el ambiente natural y con la comunidad de la cual somos parte".

\section{El enfoque de las capacidades}

El enfoque de las capacidades destaca un tipo de desarrollo relacionado a libertades, justicia, calidad de vida, dignidad y valores de las personas, en los cuales la cultura y el contexto marcan el acercamiento o el alejamiento a desempeńos, elecciones, participaciones, intercambio de valores; también, la posibilidad de generar o tener agencia, actorías sociales, reconocimiento de las capacidades y las potencialidades de los sujetos sociales para incentivar o reforzar dignidades.

Así, con estos reconocimientos y actorías sociales, las personas pueden sentirse parte de una localidad y de una comunidad grande, como puede ser la sociedad civil, e intercambiar valores universales orientados al bien común y a la justicia, pero para ello antes se deben superar la pobreza y la tiranía, e incentivar mayores libertades políticas y civiles que permitan ampliar esas capacidades en las personas. Por ello, el enfoque de las capacidades tiene unas implicancias éticas, ya que ofrece una mirada del desarrollo que busca reflexiones y comportamientos tolerantes e inclusivos desde la diversidad, donde todas las personas puedan elegir ser y hacer a partir de sus intereses personales, pero llamadas también a reconocer valores comunes, como la vida, el agua, el medioambiente, la democracia, etc.

Según Nussbaum (2012, p. 38), "el enfoque de las capacidades puede definirse provisionalmente como una aproximación particular a la evaluación de la calidad de vida y a la teorización sobre la justicia social básica. En él (en el enfoque) se sostiene que la pregunta clave que cabe hacerse cuando se comparan sociedades y se evalúan conforme a su dignidad o a su justicia básica es: ¿qué es capaz de hacer y ser de cada persona?". 


\section{Delimitando el enfoque}

El enfoque de las capacidades es un enfoque aspiracional y de proyección, que propone evaluar y analizar las elecciones que las personas realizan acerca de lo que son realmente capaces de ser y hacer, de acuerdo con una idea intuitiva de la vida que corresponde a la dignidad del ser humano. Para Nussbaum (2002, pp. 32-34), "las capacidades son universales e importantes para todos y cada uno de los ciudadanos, en todas y cada una de las naciones, y cada (persona) debe ser tratada como un fin".

Sin embargo, la misma Nussbaum (2012, p. 38) ha ampliado la definición del enfoque de las capacidades indicando que es "un enfoque comprometido con el respeto a las facultades de autodefinición de las personas. Es decididamente pluralista en cuanto a valores". Por su parte, los investigadores Phélan y Oliva (2014, p. 125) refuerzan el análisis de Nussbaum y comentan que "el enfoque de las capacidades presenta cuatro puntos importantes. Primero, cada persona como un fin en sí mismo; segundo, está centrado en la elección o en la libertad; tercero, es pluralista en cuanto a valores; cuarto y último, se ocupa de la injusticia y de las desigualdades sociales arraigadas". Para fines de nuestro análisis, es importante destacar algunos aportes al enfoque de las capacidades desde otras perspectivas:

El enfoque de las capacidades surge en el contexto del desarrollo humano. Defiende que el desarrollo no termina en el aumento de la producción económica nacional, y que por eso su estimación mediante la renta disponible es insuficiente. El desarrollo tiene que ver, más bien, con las cosas que las personas pueden realmente hacer o ser (los llamados funcionamientos), y así con las capacidades de que disponen, entendidas como las oportunidades para elegir y llevar una u otra clase de vida. Es éste el sentido en que una sociedad desarrollada es una sociedad más libre, y en el que el desarrollo es un camino hacia una libertad mayor. (Cejudo, 2007, p. 10)

En particular, el enfoque de las capacidades contribuye a la superación del subjetivismo y del utilitarismo. 
Sen ha presentado un enfoque como una superación del subjetivismo y del atomismo que caracterizan a las posturas utilitaristas, y al mismo tiempo, como una corrección aplicada a la ceguera ante las diferencias interpersonales que es inherente a las teorías de bienes o recursos.(Reyes, 2008, p. 138)

En otro aspecto, es importante destacar el aporte de este enfoque en la teoría de justicia y su puesta en práctica.

El enfoque de las capacidades humaniza, por así decir, las frías teorías contractualistas que predominan en la filosofía política, y las encamina hacia la práctica. Las teorías de la justicia deben estimular la imaginación ciudadana, salir de los recintos académicos y afrontar las circunstancias reales. (Daniel Gamper Sachse en entrevista a Martha Nussbaum en Nussbaum 2011, p. 64)

El enfoque de las capacidades pone a las personas en el centro del desarrollo y se concentra en que ellas sean sus fines; no se concentra en los medios. Ahora bien, los fines se reciben de alguien. Esto significa que las personas están inmersas en interacciones sociales e institucionales, dentro de una cultura y unos contextos, con diferentes recursos, lo cual representa también el inicio de la formación de las capacidades para establecer la posibilidad de funcionamientos alcanzables. El enfoque destaca la diversidad de las personas en contextos y culturas diferentes. A ello, se suma también el carácter universal del enfoque que permite diversas interpretaciones. Entendemos las capacidades como parte de funcionamientos de libertades auténticas, no libertades solamente instrumentales. Los fines tienen que ver con valores y principios relacionados entre sí. No son observables, pero sí perceptibles. Sen y Nussbaum indican que el ser humano debe ser capaz de elegir, y esas capacidades son las oportunidades reales de una persona de ser o hacer algo. Los fines tienen relación con "lo que se es y se hace". Por lo tanto, las personas con capacidades son sujetos sociales actuantes, que comparten con un grupo social "valores valiosos" que los mantienen unidos como personas y grupos.

Sobre el reconocimiento o la promoción del sujeto social actuante, como parte de las libertades de los sujetos sociales, consideramos que 
estas son buenas posibilidades para la proyección del enfoque, porque permite reforzar la autonomía, el autorrespeto y la autoconfianza de las personas, que las ubican en el centro del desarrollo. El planteamiento sobre las personas al centro del desarrollo humano permite una reflexión y una definición de políticas para el reconocimiento, la participación y la igualdad social en democracias inclusivas. Se actúa a partir de valores universales. También, los valores son humanos con los que las personas son tratadas con dignidad de atención, reconocidas con libertades y oportunidades. Mientras que, para Nusbaum, las capacidades están organizadas de manera específica en una lista de diez capacidades, para Sen, las capacidades no están circunscritas a una lista específica.

En el enfoque de las capacidades, se trata también de abordar las problemáticas desde el qué. Ello significa identificar cuestiones ligadas a la agencia, las potencialidades, satisfacciones, expectativas en relación con la vida que se puede llevar. Esto implica, desde el plano analítico, ver en qué enfocar las miradas, y luego preguntarse acerca del cómo se produce o genera el fenómeno o el planteamiento que se está mirando, lo cual implica que el observador debe entrenar la mirada con lecturas y trabajos prácticos para tener criterios interpretativos y evaluativos.

Para el enfoque, las capacidades humanas se dan dentro de una esfera de experiencia, es un valor compartido con otros. Es un valor, un significado. A partir de la capacidad de afiliación que comenta Nussbaum, existe una esfera social. A ello, se complementa una cuestión que para este enfoque es sustancial: el tema de la dignidad, referido a un ser racional dotado de libertad. Las capacidades se "construyen" o "constituyen" en la sociabilidad, en la cultura, no son estáticas. Por lo tanto, son mutables, lo cual requiere constantemente apelar y hacer consultas con los actores sociales que protagonizan variados desempeños en múltiples contextos. En su lista de diez capacidades humanas ${ }^{2}$, Martha Nussbaum (2002) identifica las capacidades de afiliación como de especial significado, pues estas capacidades, según

2 Las diez capacidades centrales de Nussbaum son las siguientes: 1) Vida; 2) Salud corporal; 3) Integridad corporal; 4) Sentidos, imaginación y pensamiento; 5) Emociones; 6) Razón práctica; 7) Afiliación; 8) Otras especies; 9) Juego; 10) Control del propio entorno. 
Gough (2007), se “organizan y difunden hacia los demás, para que su búsqueda sea realmente humana” (p. 184). En esta lista, Nussbaum (2002) comenta que su enfoque subraya la importancia de "una idea central del ser humano como un ser libre y digno que forma su propia vida en cooperación y recíprocamente con otros. Una vida que es realmente humana es la que está formada en su conjunto por estos poderes humanos de razón práctica y sociabilidad" (p. 84).

Propongo que ciertas normas universales de las capacidades humanas deberían ser centrales, en lo político, para las consideraciones sobre principios políticos básicos que pueden servir como soporte para una serie de garantías constitucionales en todas las naciones.

También argumentaré que estas normas se usan legítimamente para realizar comparaciones entre naciones, al indagar cómo se desenvuelven respecto a las demás en la promoción de la calidad de vida humana" [...]. El resultado que buscamos debería preservar libertades y oportunidades para cada persona, como individuos, respetándolos como fines en sí, y no como agentes o promotores de los fines de otros. (Gough, 2007, p. 179)

\section{La acción colectiva y la actoría social}

La acción colectiva se relaciona con el bien común, es decir, con la actuación en conjunto del grupo social organizado que permite la expansión de la democracia, que es el requisito fundamental para el logro de libertades y el desarrollo humano (Sen, 2000). En contextos de desigualdad social y pobreza, la acción social del grupo resulta determinante para superar estructuras que propician la subalternidad, la exclusión social y el abuso del poder. Enfocarse en el desarrollo humano y llevarlo a la práctica en contextos de este tipo pasa por reflexionar e identificar acciones sociales que amplíen libertades que permitan el logro de capacidades para el desarrollo humano.

La acción colectiva forma parte de las capacidades grupales que junto a las capacidades individuales se deben considerar para el diseño 
de una agenda política y políticas públicas. En palabras de Evans (2002), es "la acción colectiva densa, diversa, organizada [...] es necesaria para explotar las oportunidades creadas por elecciones y los derechos civiles y complementan los esfuerzos dispersos de grupos y de individuos" (p. 57). En relación con la actuación de las personas en las dinámicas colectivas, Deneulin (2006) puntualiza que "el sujeto del desarrollo humano no es el individuo aislado ni una entidad colectiva donde cada persona se vuelve indistinguible, sino que es individual y colectivo al mismo tiempo" (p. 35).

\section{Una ética para otros desarrollos}

El desarrollo se vincula cada vez más a visiones económicas y políticas respecto de la mejor calidad de vida y del uso "adecuado" de los recursos naturales. Ello implica nuevas concepciones del poder y del conocimiento dominantes. Sobre el particular, Escobar (2012) afirma que "la discusión local sobre el desarrollo no es sólo sobre el desarrollo en sí, sino sobre la historia y la cultura -sobre el Estado, la ciudadanía, las diferencia, el conocimiento, la explotación-. Se trata de la posición de las comunidades (locales) dentro del sistema mundo moderno colonial". Frente a ello, se proponen cambios de comportamiento y dejar de lado actitudes egoístas e individuales para dar paso a visiones más inclusivas y sociales.

Esto significa el cambio de una ética por otra ética, la transición de paradigmas. López Soria (2012) propone, una mirada propositiva del desarrollo, que no quede anclada en un "antimodernismo o antidesarrollismo romántico" que apunte a restaurar el mundo "premoderno" por considerarlo una especie de "paraíso perdido". "Somos hechura de la modernidad y nos toca realizar una operación de autocercioramiento crítico de nuestra realidad y asomarnos a otros horizontes de significación y de vida buena para potenciar nuestra capacidad propositiva” (López Soria, 2012, p. 6). Con ello, también se menciona que, en "América Latina se necesita recuperar la política social que pueda luchar contra los agudos niveles de pobreza que agobian a gran parte de la población, en un continente pletórico en riquezas potenciales" (Kliksberg, 2000). 
La ética que proponemos aquí tiene que ver con el bien; esto, en palabras de Carlo Maria Martini (1998), quiere decir lo siguiente:

La ética es un elemento propio del hombre, algo gracias a lo cual el hombre es lo que es. La ética establece un elemento esencial de la condición humana, que a todos afecta.

En ella emerge una esfera fundamental del significado de la vida, en el que se patentiza el sentido del límite, de los interrogantes, de la esperanza, del bien. El "bien", merece una más atenta consideración, entre otras cosas porque varias intervenciones han considerado la responsabilidad hacia el rostro de los demás como un "bien”, una elección moral justa. (Eco y Martini, 1998)

\section{Democracia participativa y ciudadanía activa}

Las democracias deben ser participativas para que las personas puedan movilizarse, sostener sus convicciones, de manera libre y crítica, pero respetando a los otros. Si no se participa de la cosa pública, pueden venir grupos autoritarios y destruir el Estado de derecho e imponer culturas de miedo o anarquía. Sin un sentido de comunidad política, difícilmente las instituciones liberales pueden funcionar. La construcción de la conciencia y de una acción colectiva ciudadana son la mejor forma de afrontar propuestas autoritarias o la corrupción. La participación es una acción colectiva que pueden contrastar, limitar o hacer frente a propuestas individuales que atentan contra el bienestar y desarrollo común.

La voluntad, la mística, el encontrar las convicciones para el actuar político ciudadano, el tener simpatía y empatía son componentes del autogobierno, que se potencian con la participación política en los foros que generan los colegios, gremios profesionales, las universidades, los sindicatos, las organizaciones de la sociedad civil; allí, en esos espacios públicos, se dan las dinámicas del consenso y el disenso, del respeto mutuo; surgen la libertad de trabajar con otros para obtener beneficios y el bien común. Los procesos de empoderamiento y de agencia son parte de la educación y del hacer de la política una actividad cotidiana. 
Entonces, por un lado, se lucha, se defienden derechos, pero también se aprende a reconocer la importancia del diálogo, del vivir en sociedad, identificando que las relaciones de poder requieren constantemente de la política y de la ciudadanía; lo contrario sería el gobierno de los autoritarios y de los que defienden intereses ajenos a los valores comunes. La ética y la ciudadanía son centrales en las repúblicas democráticas, en la formación de personas que puedan tener una opinión. La educación, las instituciones que representan los poderes del Estado, los medios deben generar esas capacidades como parte de la cultura política.

Los regímenes autoritarios o despóticos suprimen y recortan derechos, libertades e imponen culturas del miedo. Por ello, en una república donde rige el Estado de derecho y la participación como parte de un sistema democrático, la opinión, el debate, la deliberación se constituyen en prácticas de la vida de los ciudadanos. En ese sentido, el ejercicio de derechos y libertades forma parte de una democracia republicana.

\section{La participación ciudadana en la sociedad peruana}

La participación ciudadana está relacionada con acciones colectivas que demandan en general derechos en un contexto democrático, lo cual, como se ha comentado en este trabajo, es una condición para tener calidad de vida. En la pandemia de la COVID-19, en el contexto peruano, reapareció el ciudadano que invoca un estado más presente en su vida social ${ }^{3}$. Esta invocación representa el vínculo por gobierno en el que el valor público debe estar centrado en la calidad y alcance de los servicios públicos hacia los ciudadanos. Esta situación evidencia también la necesidad de un sistema que articule políticas, enfoques y especialistas en políticas públicas para crear sistemas y manejar sistemas. Sin políticas (que incluyan valores ciudadanos), al igual que múltiples

3 El sentido de la reaparición del ciudadano en la vida pública no es retórico, sino real. Ello se ha constatado entre el 2016 y el 2019 en observatorios de medios en el que los ciudadanos no tienen voz activa en las noticias de la prensa de Lima. Observatorio Universitario de Medios - Fundación Mohme - Escuela de Periodismo - Universidad Antonio Ruiz de Montoya. 
miradas para atender los diferentes escenarios de la problemática del país, como, por ejemplo, la falta de servicio de agua potable o la escasa bancarización de la población, se puede gestionar, pero no tener sistemas adecuados para la atención.

Desde nuestro enfoque, el paso previo para reducir la pobreza está en proyectar sistemas más inclusivos y gestionarlos con transparencia y rendición de cuentas. La crisis de la COVID-19 ha develado que puede haber capital económico y gestión, pero sin sistemas localizados y focalizados a los contextos y realidades del país, esa gestión es limitada.

Se necesitan sistemas más justos para afrontar dificultades reales, que en el país se dan cada cierto tiempo, como los terremotos y los desastres naturales ocasionados por el fenómeno del Niño. Estos eventos han llevado a gran parte de la población a la pobreza, y a vivir situaciones de desigualdad social. Los otros problemas estructurales que tienen efectos en la desigualdad social han sido la falta de planificación urbana de las ciudades, la violencia política interna y los delitos ambientales relacionados en parte a los conflictos socioambientales y a la extracción de recursos naturales de manera ilegal, que ejercen serios impactos negativos en las personas y el medio ambiente. En estos procesos sociales, políticos y económicos que han desencadenado situaciones de pobreza y desigualdad, el efecto de la COVID-19 ha tenido serios impactos, debido a la falta de atención o a las limitaciones en el acceso al bienestar. Sin embargo, destacamos la acción colectiva de las personas para afrontar estas adversidades.

\section{Acciones colectivas en el contexto del COVID-19}

Entre las acciones colectivas, podemos mencionar, en el contexto peruano, las siguientes iniciativas, sin ser exhaustivos para contrarrestar la pandemia de la COVID-19: a) la organización de las ollas comunes en zonas urbano-marginales de Lima Metropolitana (La República, 2020), para superar el hambre y la desnutrición que ofrecen una dieta completa con proteínas que la entrega de canastas no cubre, porque incluye sobre todo carbohidratos; b) las campańas de solidaridad en Loreto para implementar con una planta de oxígeno medicinal el 
hospital regional (Agencia Andina, 2020) que recibió pacientes con COVID-19, c) el "Comando Matico", una iniciativa de indígenas shipibo-konibo (AIDESEP. 2020) que incluye tratamientos paliativos a indígenas amazónicos contagiados con COVID-19, d) asociaciones de comerciantes de mercados de abastos (Agencia EFE, 2020), los cuales generaron estrategias para la comercialización de productos ante la falta de orientación sobre el manejo de protocolos anti-COVID-19, e) la emisión de programas de radio en lenguas originarias, como el quechua y las lenguas amazónicas (SIGNIS ALC, 2020), para ofrecer orientación e información sobre la COVID-19.

En los casos que se mencionan aquí, se identifican acciones colectivas relacionadas a mitigar el hambre, afrontar la falta de oxígeno medicinal, hacer efectiva la atención médica intercultural, la importancia de la orientación y la capacitación en comerciantes de mercados de abastos a nivel nacional, al igual que una programación informativa y noticiosa con pertinencia intercultural. Podemos ver que estas acciones colectivas han incidido en la calidad de vida de la población en desventaja social por la falta de información, alimentación adecuada, componentes medicinales y atención médica de tipo intercultural.

\section{Acciones colectivas por derechos y el medioambiente}

Sin embargo, en Perú, existen otras experiencias valiosas relacionadas a la participación colectiva, que tienen su inicio antes de la pandemia, vinculados con los servicios de justicia, acceso al agua y el desarrollo del hábitat ambiental, como los casos de las rondas campesinas (Defensoría del Pueblo, 2006), las comunidades andinas que cosechan el agua ${ }^{4}$ o la experiencia de forestación de los andes con miles de pinos ${ }^{5}$. A todo ello, también se suman las movilizaciones y las protestas sociales para luchar

4 La recuperación de las prácticas de siembra y cosecha de agua en Ayacucho como medida de adaptación al cambio climático y la seguridad hídrica (Rossi Taboada, 09/12/2018).

5 Carton, C. (1996). Reforestación y manejo de cuencas en la CAT. Atahualpa Jerusalén. Granja Porcón, Cajamarca. En Adefor, Manejo integral de microcuencas. Jequetepeque - Cajamarca (pp. 185-195). 
contra la corrupción, la reivindicación de derechos para los jóvenes trabajadores, los indígenas amazónicos y los derechos de la mujer. Estas movilizaciones indican que los canales de comunicación y los espacios de deliberación no se dieron, o se agotaron, en la búsqueda de justicia y verdad. Las manifestaciones que se recuerden para reivindicar derechos y libertades ciudadanas han sido las siguientes: la "marcha de los cuatro suyos" (2000), "El Baguazo" (2009), el conflicto minero de Conga (2012), las protestas contra la "Ley Pulpín"(2014), El conflicto minero de "Tía María” (2015), "Ni una menos" (2016), "Marchas contra la corrupción" (2019) y "Las marchas para el cierre del Congreso de la República” (2019).

Al respecto, Walzer (1994) denomina a la actitud de defensa de los derechos esenciales de la verdad y la justicia como "discurso moral minimalista”.

No hay muchas cosas que sean más importantes que la "verdad" o la "justicia”, entendidas minimalísticamente. Las demandas mínimas que nos hacemos unos a otros, si se nos niegan, son reiteradas con insistencia y pasión. En el discurso moral, la delgadez y la intensidad caminan juntas, mientras que con la densidad caminan la cualificación, el compromiso, la complejidad y el desacuerdo. La experiencia de la búsqueda de la verdad y la justicia está relacionada con un discurso moral minimalista, llevado al terreno de las experiencias circunstanciales e históricas. La densidad tiene que ver con una moralidad en el núcleo de la experiencia social, que es densa, compleja, requiere o demanda cualificación y donde hay desacuerdo. (Walzer, 1994, p. 38)

\section{Consideraciones finales}

Siguiendo con nuestro planteamiento inicial, proponemos que relacionar el concepto de calidad de vida con el enfoque de las capacidades permite situar la teoría del desarrollo humano en las personas, contextos y culturas, particularmente en el contexto de los pobres. Desde este planteamiento, se destaca para el análisis cuestiones claves sobre el desarrollo humano, como el hecho de tener bienestar 
y participar como parte del ejercicio de libertades y derechos en la vida democrática. La ausencia o la limitación al acceso de estos valores democráticos representa desafíos para que los encargados de las políticas públicas, las instituciones de la opinión pública y los educadores orienten acciones hacia los más vulnerables, en un acto de humanidad en la crisis de la COVID-19, pero también para consolidar la comunidad democrática.

Por otro lado, el enfoque de las capacidades pone el foco en los problemas sociales, que concierne a todos; También para superar estereotipos y prejuicios contra grupos sociales (como los migrantes o los indígenas). Se debe evitar encasillar y limitar excesivamente el debate en culturas (que puede exacerbar chauvinismos y malentendidos tribales) $y$, en cambio, podemos dirigir el debate hacia la importancia de la educación y la justicia (Appiah, 1998), para que todos puedan recibir por igual. El gran trabajo de reconstrucción para salir de la crisis de la COVID-19 consiste en evaluar, concebir y establecer vínculos posibles entre el Estado y los ciudadanos. Los casos que hemos presentado aquí como acciones colectivas permiten ver la confluencia de valores y necesidades, que pueden ser buenas prácticas para el inicio de procesos inclusivos y políticas públicas. 


\section{Referencias}

Agencia Andina (2020). "Coronavirus: ciudad de Iquitos contará con planta de oxígeno propia”, 4 de mayo 2020. Recuperado de: https://andina.pe/agencia/noticia-coronavirus-ciudad-iquitoscontara-planta-oxigeno-propia-795820.aspx

Agua-Andes (2018). "La recuperación de las prácticas de siembra y cosecha de agua en Ayacucho como medida de adaptación al cambio climático y la seguridad hídrica”. Rossi Taboada 09/12/2018. Recuperado de: https://www.agua-andes.com/ articulos/recuperacion-practicas-siembra-y-cosecha-aguaayacucho-como-medida-adaptacion-al-cambio

Agencia EFE (2020). "Mercados de Perú quieren quitarse el estigma de ser foco de contagio de COVID”, 19 de mayo 2020. Recuperado de: https://www.efe.com/efe/america/economia/ mercados-de-peru-quieren-quitarse-el-estigma-ser-fococontagio-covid-19/20000011-4250441

AIDESEP (2020). "Ucayali: Jóvenes indígenas atienden a su pueblo en tiempos de COVID-19", 16 de mayo 2020. Recuperado de: http://www.aidesep.org.pe/noticias/ucayali-jovenes-indigenasatienden-su-pueblo-en-tiempos-de-covid-19

Ardila, Rubén (2003). "Calidad de vida: una definición integradora”, en: Revista Latinoamericana de Psicología, vol. 35, núm. 2, 2003, pp. 161-164. Fundación Universitaria Konrad Lorenz. Colombia.

Banco Mundial (2020). "Crisis por el coronavirus aumentó las desigualdades en el Perú”, 8 de setiembre 2020. Recuperado de: https:/www.bancomundial.org/es/news/press-release/2020/ 09/08/crisis-por-el-coronavirus-aumento-las-desigualdades-enel-peru 
Carton, Charles (1996). "Reforestación y manejo de cuencas en la CAT. Atahualpa Jerusalén. Granja Porcón, Cajamarca”, en Adefor, Manejo integral de microcuencas. Jequetepeque - Cajamarca (pp. 185-195).

Cejudo, Rafael (2007). “Capacidades y libertad Una aproximación a la teoría de Amartya Sen”, en Revista Internacional de Sociología (RIS), Vol. Lxv, No 47, Mayo-Agosto, pp. 9-22.

Deneulin, Séverine (2006). Necessary Thickening: Ricoeur's Ethic of Justice as a Complement to Sen's Capability Approach". Deneulin et al. (eds.). Transforming Unjust Structures. The Capability Approach, Dordrecht. Springer.

Defensoría del Pueblo (2006). "El reconocimiento estatal de las rondas campesinas compendio de normas y jurisprudencia". Recuperado de: https://www.defensoria.gob.pe/modules/ Downloads/informes/varios/2005/rondas_campesinas.pdf

Eco, Umberto; Martini, Carlo (1998). “¿En qué creen los que no creen?” Argentina: Editorial Planeta. Recuperado de: http:// www.mercaba.org/ARTICULOS/E/en_que_creen_los_que_ no_creen.htm

Escobar, Arturo (2012). "Más allá del desarrollo: postdesarrollo y transiciones". En: Revista de Antropología Social, 21, pp. 23-62.

Evans, Peter (2002). "Collective Capabilities, Culture and Amartya Sen's Development and Freedom". Studies in Comparative International Development, XXXVII, 2, pp. 54-60.

Gough, Ian (2007). "El enfoque de las capacidades de M. Nussbaum: un análisis comparado con nuestra teoría de las necesidades humanas". En: Papeles de Relaciones Ecosociales y Cambio Global, $\mathrm{N}^{\circ}$ 100, CIP-Ecosocial/Icaria, invierno 2007/08. Madrid, traducción: Leandro Nagore y Silvina Silva, pp. 177- 202. 
La República (2020). "Las ollas comunes para la emergencia alimentaria”, 1 de octubre de 2020. Recuperado de: https:// larepublica.pe/opinion/2020/10/01/las-ollas-comunes-para-laemergencia-alimentaria-la-periferia-es-el-centro/

Lopez Soria, José (2012). "Rio + 20 y el desarrollo", en Bernex, N. y A. Castro (ed.) (2015). Rio +20 Desafíos y perspectivas. Lima: INTE/ PUCP.

Nussbaum, Martha (2002). Las mujeres y el desarrollo humano. El enfoque de las capacidades. Barcelona: Herder.

(2011). Libertad de conciencia: el ataque a la igualdad de respeto. "Vivir en democracia implica respetar el derecho de las personas a elegir estilos de vida con los que no estoy de acuerdo" (entrevista de Daniel Gamper Sachse). Buenos Aires: Katz editores.

humano.

(2012). Crear capacidades. Propuestas para el desarrollo

Barcelona: Paidós.

Reyes, Agustín (2008). "Comunidades de significación como capacidades colectivas. Una revisión comunitarista de la teoría de Amartya Sen”. ARETÉ Revista de Filosofía Vol. xx, N 1, pp. 137-163.

Sen, Amartya (2000). Desarrollo y Libertad. Madrid: Editorial Planeta.

SIGNIS ALC (2020). "La comunicación indígena durante el coronavirus.", 23 de abril de 2020. Recuperado de: http://signisalc.org/noticias/ amazonia/pueblos-indigenas/23-04-2020/la-comunicacionindigena-durante-el-coronavirus

Walzer, Michael (1994). Moralidad en el ámbito local e internacional. Alianza Editorial. 